\title{
THE CLEMENTINE ATTITUDE DETERMINATION AND CONTROL SYSTEM
}

\author{
Paul DeLaHunt, Steve Gates, Marv Levenson \\ U. S. Naval Research Laboratory \\ Glenn Creamer \\ Swales \& Associates
}

\begin{abstract}
The principle objective of the Clementine spacecraft was to test the most advanced lightweight imaging sensors and component technologies for the next generation of Department of Defense spacecraft. As a secondary mission, Clementine was to obtain scientific data from a two month lunar mapping mission and a fly-by of the near-Earth asteroid 1620 Geographos. To meet these objectives the Attitude Determination and Control System (ADCS) was responsible for maintaining attitude knowledge and control in Earth and Lunar orbit, during the fly-by of Geographos, and during all the orbit transfers. This paper will present a description of the ADCS architecture, identify hardware selected, and show how the hardware and software was tested. ADCS hardware consisted of all new lightweight spacecraft components including two three-axis attitude determination Star Tracker Cameras, an Interferometric Fiber Optic Gyro Inertial Measurement Unit, a Ring Laser Gyro Inertial Measurement Unit, and a set of four Reaction Wheels, with a total system weight of less than $13 \mathrm{~kg}$.
\end{abstract}

\section{Introduction and Mission Overview}

Clementine, the Deep Space Program Science Experiment spacecraft developed by the Naval Research Laboratory (NRL) and cosponsored by the Ballistic Missile Defense Organization (BMDO) and NASA, was launched from Vandenberg Air Force Base on January 25, 1994 using a Titan IIG launch vehicle. The Clementine spacecraft was designed, built, tested, and operated by NRL's Naval Center for Space Technology. Clementine's primary objective was to space-qualify advanced lightweight imaging sensors and component technologies during its mission to lunar orbit and its fly-by of the nearEarth asteroid Geographos. The Goddard Space Flight Center and the Jet Propulsion Laboratory assisted NRL in orbit determination and maneuver planning throughout the duration of the mission. The Lawrence Livermore National Laboratory (LLNL) provided small, lightweight cameras and mission sensors to record high resolution images in a wide spectrum of wavelengths. Included in the sensor package were two star tracker cameras (ST), an ultraviolet/visible camera (UV/Vis), a near infrared camera (NIR), a long wave infrared camera (LWIR), a high resolution camera (HiRes), and a laser image detection and ranging camera (LIDAR). Specific characteristics of the sensor payload as well as general descriptions and objectives of the Clementine mission are provided in References 1 and 2.

Clementine achieved lunar orbit on February 21, 1994 through a series of multiple phasing loops and spent approximately two months in two separate lunar orbits, during which time it successfully mapped the entire surface of the moon in ultraviolet/visible and near-infrared spectrums. Clementine was then scheduled to travel towards Geographos after entering a highly elliptical orbit about the earth with a lunar gravity assist. However, on May 7, 1994 an on-board computer malfunction inadvertently activated several jet thrusters, expelling the remaining attitude control fuel. As a result, the asteroid flyby phase of the mission could not be achieved. A complete description of the nominal Clementine mission astrodynamics is provided in Reference 3.

The NRL/Clementine effort began January 1992. A Preliminary Design Review (PDR) was held at six months and the Critical Design Review (CDR) was held at ten months. Attitude Determination and Control System (ADCS) component procurement was initiated prior to the PDR with contracts put in place at the time of the CDR. Components were delivered around nineteen months with launch at twenty-three months into the program.

In this paper we describe the ADCS architecture, how it was operated, identify hardware selected, and describe how the hardware and software was tested. Descriptions of the vehicle configurations, payload sensors, and spacecraft characteristics are provided in section II. The attitude determination, spin stabilization control, and three-axis control systems will be described in section III. Section IV will describe 
the ADCS components and section $\mathrm{V}$ will discuss hardware and software testing. Finally, some concluding remarks will be given in section VI.

\section{Spacecraft Configuration and Characteristics}

The Clementine spacecraft had two primary vehicle configurations during the mission. The spacecraft was in the configuration as shown in Figure 1 until the Solid Rocket Motor (SRM) burn for low Earth orbit (LEO) departure. The spacecraft was three-axis stabilized for the one week checkout period prior to being spin stabilized for 4 hours in preparation for the 63 second SRM trans-lunar injection burn. In this configuration the spacecraft is dominated by the SRM which is attached and fully loaded, and the two solar array panels are in their stowed position. After completion of the SRM burn the spacecraft is reconfigured to the configuration as shown in Figure 2. In this configuration the spacecraft is three-axis stabilized with the two solar array panels deployed and the SRM separated. The vehicle maintains this configuration throughout its mission. There were two intermediate configurations prior to the final separation of the empty SRM, the first with the solar array panels stowed and the second with the solar array panel deployed. These intermediate configurations were only used for a few hours during spin-down after the SRM burn and during initial checkouts of the payload sensors. The spacecraft mass and inertias for the first (or LEO) configuration and second (or Deployed) configuration are provided in Table 1.
The science payload masses are provided in Table 2 and some additional spacecraft characteristics are given in Table 3 .

Table 1: Clementine Mass and Inertia Properties

\begin{tabular}{l|c|c|c}
\hline & $\begin{array}{c}\text { LEO } \\
\text { Config } \\
\text { (Wet) }\end{array}$ & $\begin{array}{c}\text { Deployed } \\
\text { Config } \\
\text { (Wet) }\end{array}$ & $\begin{array}{c}\text { Deployed } \\
\text { Config } \\
\text { (Dry) }\end{array}$ \\
\hline Mass (Kg): & 1647 & 456 & 233 \\
\hline Inertias (Kg-m²) & 199 & 93 & 66 \\
Ixx: & 733 & 80 & 56 \\
Iyy: & 736 & 107 & 77 \\
Izz: &
\end{tabular}

Table 2: Science Payload Masses

\begin{tabular}{l|c}
\hline \hline Science Payload & Mass (kg) \\
\hline Star Tracker Cameras (2) & 0.3 \\
UV/Visible Camera & 0.5 \\
Near IR Camera & 1.9 \\
Long Wave IR Camera & 1.8 \\
High Resolution Camera & 1.3 \\
Laser Transmitter & 0.6 \\
Charged Particle Telescope & 0.2 \\
Dosimeters (4) & 0.1 \\
Radiation Experiment & 0.7 \\
Orbital Meteoroid and Debris & 0.6 \\
$\quad$ Counting Experiment & \\
\hline Total & 8.0 \\
\hline \hline
\end{tabular}

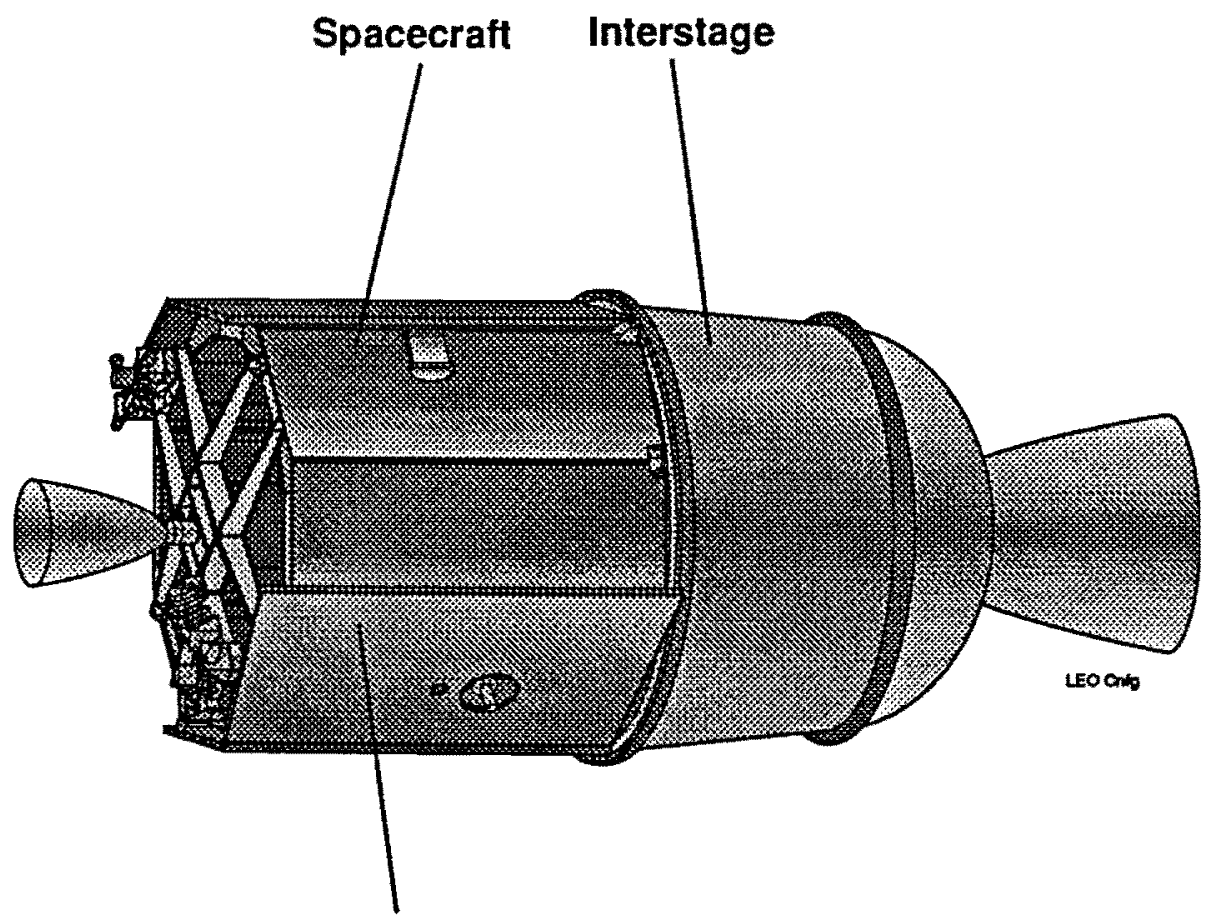

Solar Array Panels

Figure 1: Spacecraft Configuration In Low-Earth Orbit 
Table 3: Spacecraft Characteristics

\begin{tabular}{|c|c|}
\hline Mass: & $-456 \mathrm{Kg}$ (wet) \\
\hline Power: & -360W@1AU \\
\hline Size: & $-1.14 \mathrm{~m}$ diam $\times 1.88 \mathrm{~m}$ length \\
\hline Characteristics: & $\begin{array}{l}\text { - Three-axis stabilized } \\
\text { - Single Axis Gimbaled GaAs } \\
\text { solar arrays } \\
\text { - Fixed } 1.1 \mathrm{~m} \text { high-gain antenna } \\
\text { - Fixed payload sensors } \\
\text { - Combined active and passive } \\
\text { thermal design }\end{array}$ \\
\hline Telemetry: & $\begin{array}{l}\text { - S-Band RF link to NASA } \\
\text { DSN and DoD tracking stations }\end{array}$ \\
\hline Data Rate: & $\begin{array}{l}\text { - Downlink selectable between } \\
0.125 \text { and } 128 \mathrm{~kb} / \mathrm{s}\end{array}$ \\
\hline $\begin{array}{l}\text { On-Board } \\
\text { Storage: }\end{array}$ & - 1.9 Gbit solid state recorder \\
\hline Propulsion: & $\begin{array}{l}-4865 \mathrm{~m} / \mathrm{s} \Delta \mathrm{V} \\
\text { - } \mathrm{Bi}-\mathrm{Propellant}(\mathrm{MMH} \& \\
\mathrm{N}_{2} \mathrm{O} 4 \text { ) } 489 \mathrm{~N} \Delta \mathrm{V} \text { Motor } \\
\text { - Mono-Propellant (Hydrazine) } \\
\text { with ten } 5.3 \mathrm{~N} \text { and two } 22.7 \mathrm{~N} \\
\text { ACS thrusters } \\
\text { - STAR 37FM Kick Motor }\end{array}$ \\
\hline
\end{tabular}

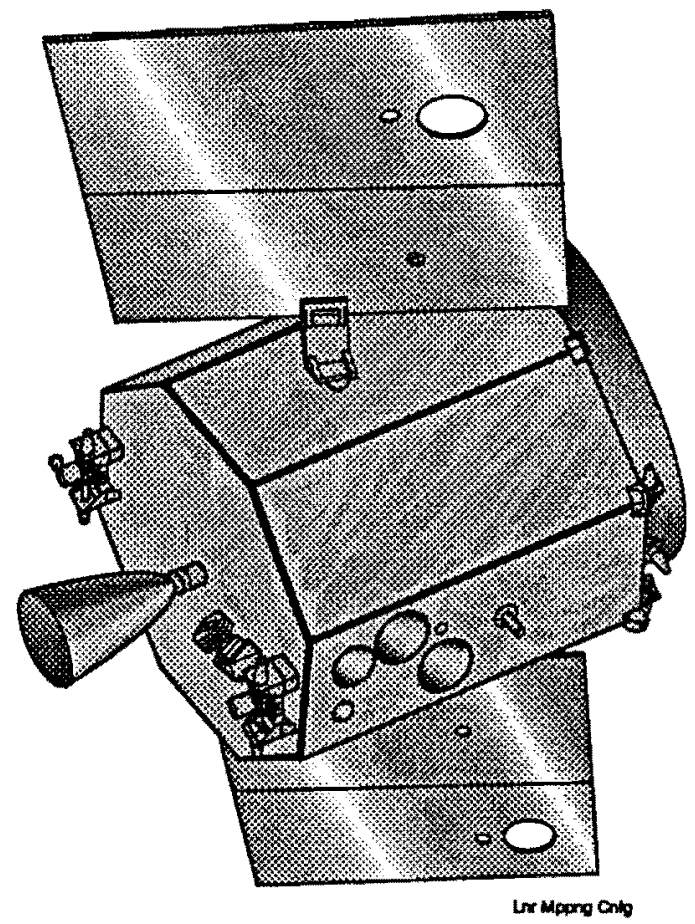

Figure 2: Spacecraft Configuration During Lunar Mapping

\section{Attitude Determination and Control Subsystem}

The primary requirements for Clementine were to test advanced lightweight sensors and technologies developed by the BMDO and industry and to collect as much science data as possible from a lunar mapping orbit and a fly-by of a near-earth asteroid in less than two years with stringent budget and weight restrictions. To meet this objective, the spacecraft and primary attitude control modes that evolved were as follows. During LEO the vehicle was three-axis stabilized using a bang-bang thruster control system. After the one week spacecraft checkout period, the vehicle was spun-up, as a prolate spinner, for approximately 4 hours for execution of the SRM burn to put the spacecraft into a phasing loop trajectory to the moon. Shortly after the SRM burn, the vehicle was spun-down and returned to three-axis bang-bang thruster control. The solar panels were deployed and the SRM was separated to put the spacecraft into its final fully deployed configuration. Once in its final configuration, control was switched to a mode using the three orthogonal reaction wheels, with a fourth skewed wheel as a back-up. Clementine remained in this control mode throughout the mission with the exception of $\Delta V$ burns when the control system was switched back to three-axis bang-bang control using the thrusters.

\section{Attitude Determination}

For Clementine, the attitude knowledge requirement was 3 degrees in LEO to meet the pointing requirements of the SRM burn, 0.25 degrees during $\Delta V s$, and 0.03 degrees for image data collection. To meet the tight requirement of image data collection during cruise to the moon, lunar mapping, and cruise to and fly-by of the asteroid, it was necessary to use a combination of star trackers and gyros. Typically this configuration of sensors is made up of high accuracy narrow field of view star trackers and high accuracy low drift gyros with the addition of coarse attitude sensors for initial acquisition. However for this mission, with its weight and schedule limitations, an alternate configuration of star trackers and gyros was needed. A new BMDO/LLNL developed lightweight ST camera was used in combination with two $1 \mathrm{deg} / \mathrm{hr}$ class gyro packages.

The ST camera is capable of full three-axis attitude determination from any attitude which eliminated the need for additional coarse attitude sensors for attitude acquisition. Clementine's two inertial measurement units (IMUs) provide 
redundant three-axis vehicle rotational information in the form of delta-angles which are used to generate the three-axis rate signal for quaternion propagation. The ST camera is used to compensate for the gyro rate biases in the IMUs. This is accomplished by combining both the IMUbased attitude quaternion and the ST-based attitude quaternion into a simple fixed-gain Kalman filter. The function of the IMU/ST filter was to combine two sensor outputs so as to provide an updated attitude estimate as well as an estimate of the gyro rate biases along each axis. Biases are canceled from the IMU signals to improve the quality of the quaternion propagation between ST measurements.

\section{Spin Stabilization}

To perform the SRM burn, Clementine was spun-up to $60 \mathrm{rpm}$ about its minimum inertia axis making it a prolate spinner. Four modes of operation were available during the spin phase. These consisted of Active Nutation Control (ANC) for controlling nutation angle growth, Spin Axis Precession (SAP) for correction of spin axis pointing errors, Closed Loop Spin Rate Control (CLSRC) for any final corrections of spin rate, and Open Loop Spin Rate Change (OLSRC) to perform desired open loop commanding of the attitude control system thrusters.

The spin-up sequence started with an OLSRC to an intermediate spin rate of $30 \mathrm{rpm}$. The vehicle remained at this spin rate for the majority of the spin phase to reduce the fuel consumption while performing ANC. Once at 30 rpm, ANC was used to reduce any initial nutation angle built up during the spin-up. After driving nutation angle down, the SAP mode was available if a significant pointing error had developed during spin-up. ANC was then commanded on and left active until shortly before the final spinup to $60 \mathrm{rpm}$ which was also performed using the OLSRC mode. A short period of time was available to perform ANC and CLSRC prior to committing to the SRM burn. There was no effort to control either nutation angle or precession angle during the SRM burn. Spin-down was performed using the OLSRC, ANC, and CLSRC. Once at zero spin rate, the control mode was returned to three-axis bang-bang control using the thrusters.

ANC was designed to use spacecraft inertia estimates and IMU delta theta outputs to compute and monitor the transverse rates and nutation angle. When the nutation angle and transverse rate exceeded pre-selected limits, one of two $5 \mathrm{lbf}$ thrusters was fired to drive the transverse rates, and as a result the nutation angle, back down. The nutation angle limit, the transverse rate deadband, and thruster size were selected to maintain control over a vehicle with a very low energy dissipation time constant and to minimize momentum vector walking. ANC was configured to initiate a nutation thruster burn when the nutation angle exceeded an upper limit and transverse rate was out of the deadband. If the deadband had been selected too low there would have been a significant amount of momentum vector walking due to the relatively large thrusters used. To minimize momentum vector walking, the deadband was widened to limit the nutation thruster pulse to the minimum impulse bit of the thruster system.

The SAP mode was available to correct spin axis pointing errors incurred during either spin-up events or errors due to momentum vector walking when in ANC. The errors incurred during spin-up are primarily due to thruster misalignment, dynamic balance capability, and initial pointing errors and transverse body rates at start of spin-up. The SAP logic required knowledge of the vehicle three-axis attitude for timing the precession pulses.

The IMU was used to propagate the threeaxis attitude after a final attitude initialization from the ST camera prior to spin-up. Knowledge of the pointing error magnitude was limited by angular random walk and scale factor errors of the IMU. Knowledge of the orientation of the vehicle about the spin axis, or roll orientation, rapidly degraded due to scale factor errors in the IMU. As the roll orientation degrades the knowledge of the direction to precess degrades to the point that performing an SAP could increase the true error. The roll orientation was still accurate enough right after spin-up that SAP would be successful without any roll updates. To prolong the ability to perform an SAP, an external reference was required to update the roll orientation. This was provided by the solar array auto-track system which contained two sun sensors. These sensors could provide a crude measure of sun position through the voltage level as the sun passed across its surface. Peak detection logic was used to determine the time that the sun crossed the plane containing the spin axis and the normal to the sun sensor.

When the SAP command is sent the logic on-board determines the proper timing for thruster pulses to precess the spin axis from the current pointing vector to the desired pointing vector. Knowledge of the angle between the spin axis and the desired $\Delta \mathrm{V}$ pointing vector was on the order of one degree with a control requirement of five 
degrees during the SRM burn. Roll knowledge was only required to less than ten degrees.

As a result of roll attitude error and the angular random walk component of the gyros, it was desirable to limit use of the SAP logic to early in the spin-up. During spin-up from $30 \mathrm{rpm}$ to $60 \mathrm{rpm}$, the stiffness of the spin axis would be such that there would be little pointing error growth.

\section{Three-Axis Stabilization}

When in three-axis attitude control, the system was in one of six guidance modes and one of two control modes. The six guidance modes consisted of Earth Pointing, Lunar Mapping, Star Pointing, Inertial Pointing, Star Tracking, and Terminal Guidance. The two control modes used either reaction wheels or thrusters as actuators. The thruster control mode was used for all $\Delta \mathrm{Vs}$ and was to be used for the asteroid fly-by. The reaction wheel control mode was used for all other operations. The inputs to the six guidance modes varied by mode, but the output of all six consisted of a series of commanded attitude quaternions and body rates. The output of the two control modes consisted of commands to the reaction wheels or thrusters. The top level combination of the guidance and control modes with the attitude determination sensors is shown in Figure 3. The solid lines represent measurable or calculated signals and the broken lines represent unknown signals.

In Earth Pointing mode, the spacecraft uses the on-board ephemeris to compute the proper attitude quaternion for pointing the spacecraft's high gain antenna to the center of the earth or to a selectable ground station. This mode was used to transmit the images collected during lunar mapping. When commanded to perform Lunar Mapping, the spacecraft would use the onboard ephemeris to compute the commanded quaternion and body rates for tracking lunar nadir with the payload sensor line of sight. An option was available to add a small cross-track offset as part of the command to enter the mode. A star catalogue was stored on-board to allow the Star Pointing mode to be commanded to slew the payload sensor line of sight to a particular star. The Inertial Pointing mode allowed a single quaternion with zero body rates or a table of quaternions and body rates to be sent up to the control system. It was with this mode that certain points, such as the Apollo landing sites, could be tracked or special scan profiles could be performed. The two final modes, Star Tracking and Terminal Guidance both used feedback from the payload sensors to adjust the commanded attitude to maintain track on a star, earth or moon limb, or the asteroid.

The primary control mode for Clementine used a PID control law, with reaction wheels as the primary vehicle actuator. This control mode was used for all guidance modes except when performing the final approach and fly-by of the asteroid and during $\Delta \mathrm{V}$ maneuvers when the reaction wheel torque capability was insufficient to counter thrust misalignments. Each axis was treated as independent with any cross-axis coupling treated as a disturbance source. Any constant (or nearly constant) disturbances such as

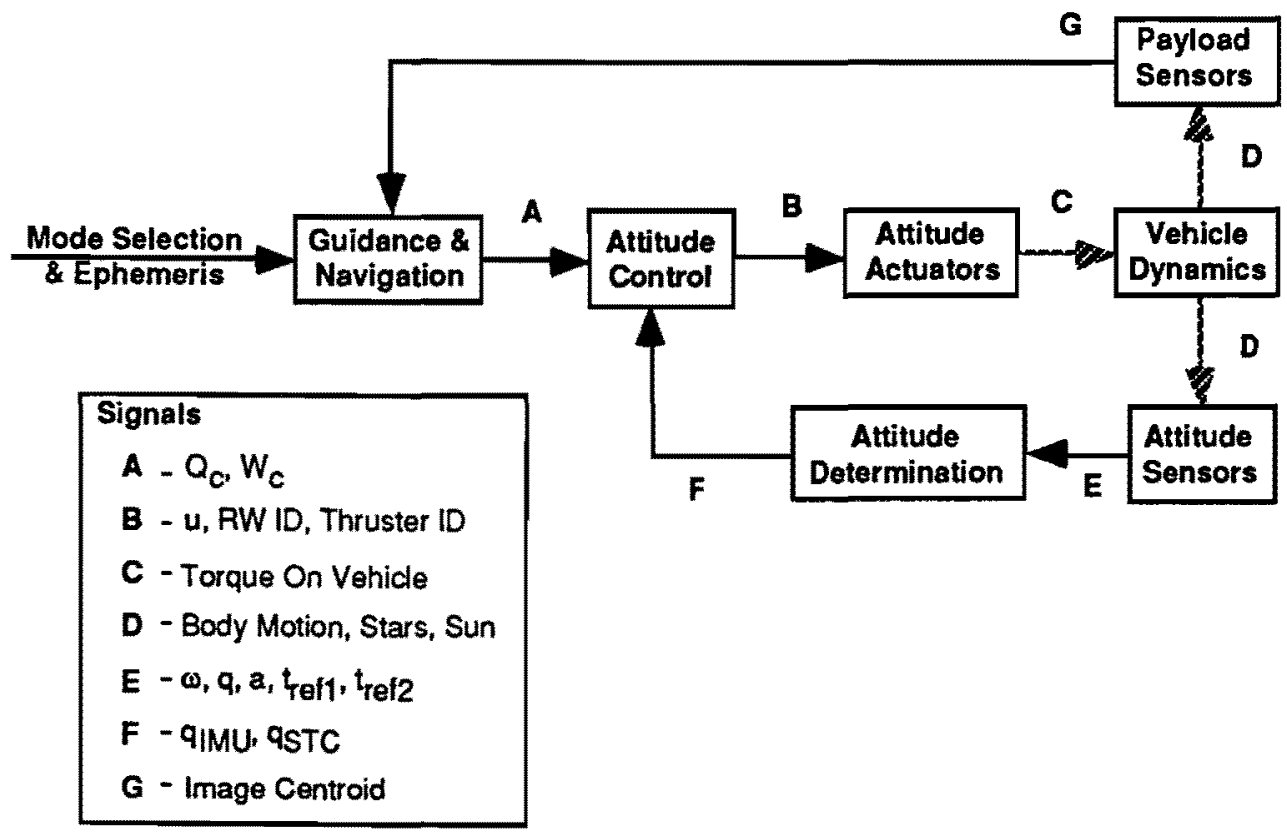

Figure 3: Attitude Determination \& Control System Block Diagram 
reaction wheel friction were handled with the small integral feedback term.

Large angle slew logic was required to smooth transitions between modes. Whenever the ADCS software received a guidance mode transition command, the new commanded attitude quaternion was compared with the current estimated attitude quaternion to determine if the change was greater then a pre-selected limit. If true, then a large angle rest-to-rest slew maneuver was performed. The logic for large angle slews computed the angle of rotation of the vehicle about a skewed eigenaxis. The logic first computed the eigenangle and eigenaxis of rotation that would take the vehicle from its current quaternion to the desired quaternion. When using the reaction wheel control system a commanded bang-coast-bang time history of the slew was then determined such that the wheel speeds and the applied torques stayed within prescribed bounds. When using the thruster control system, the slew was performed using a rate command for each axis. The eigenaxis rate command was based on a maximum pulse time for any given large angle slew. The three body rate commands are then computed to slew the vehicle about the eigenaxis.

Clementine required $134,000 \mathrm{lbf}-\mathrm{sec}$ of total impulse to meet the mission trajectory requirements. This was met using a bi-propellant reaction control system using $\mathrm{MMH}$ and $\mathrm{N}_{2} \mathrm{O}_{4}$. The motor selected had a thrust of $110 \mathrm{lbf}$. To control the vehicle throughout the various burns, a bang-bang hydrazine thruster control system was used. The reaction wheels could not provide the torque required to counter thrust misalignment associated with the motor.

The hydrazine thruster control system was driven by a proportional plus derivative control law and used all available thrusters. The thruster configuration is shown in Figure 4. In the LEO configuration, only the six $+X$ thrusters were available. The other six were under the SRM/Interstage Adaptor. An option was available to select between a minimum thruster set and all thrusters. With this, fuel could be conserved when control authority was not a concern. In Figure 4, VJ is the $110 \mathrm{lbf} \Delta \mathrm{V}$ thruster, P1 and P2 are both $5 \mathrm{lbf}$ thrusters and the other ten thrusters have $1 \mathrm{lbf}$ of thrust. The attitude control thrusters used a blow-down hydrazine system. The thrust from the two $5 \mathrm{lbf}$ engines decreased to $2.1 \mathrm{lbf}$ and the ten $1 \mathrm{lbf}$ thrusters decreased to $0.4 \mathrm{lbf}$ as the hydrazine was consumed.

The drop in thrust level presented a possible problem for worst case misalignments of the $110 \mathrm{lbf}$ motor at end of life. An analysis and industry survey was performed to determine a

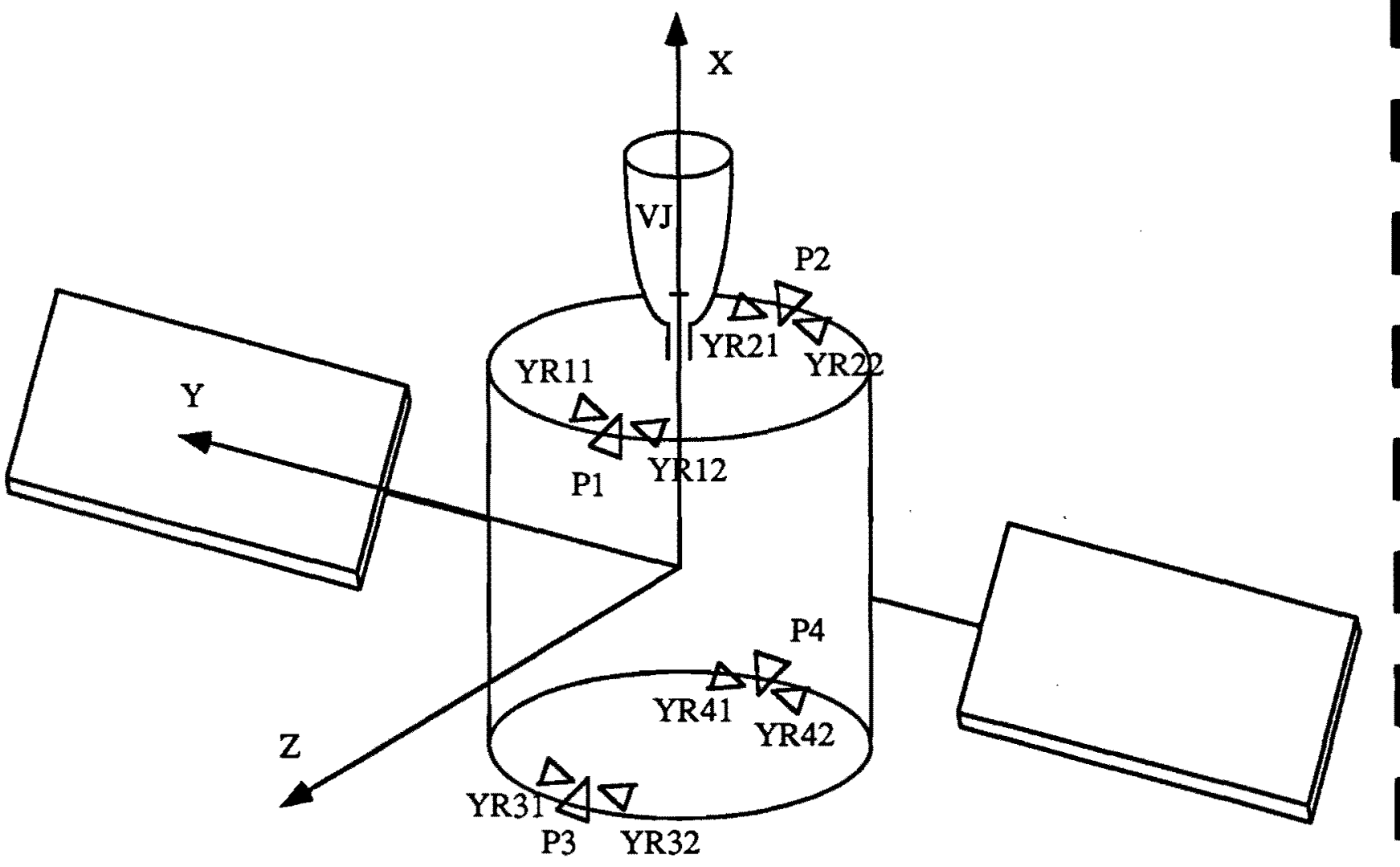

Figure 4: Attitude Control System Thruster Configuration 
worst case estimate of the thrust misalignment for the $110 \mathrm{lbf}$ motor. The majority of motor users assumed large errors which would in our case result in loss of control authority at the end of mission. All of these motor users either use the motor while spinning or as one of a group of motors, so the errors do not contribute significantly. However, one other motor user uses significantly smaller values for misalignment, that would allow for sufficient control authority at the end of mission for our configuration. With the support of a review and evaluation of the possible error sources we chose to use the values of 0.1 degrees for angular misalignment and 0.12 inches for offset. Spacecraft alignment and balance requirements were specified such that all other misalignment sources would be negligible when compared with the motor thrust misalignment.

The asteroid fly-by phase of the Clementine mission was designed such that the vehicle tracks the near-Earth asteroid Geographos with a closest approach distance of $100 \mathrm{~km}$. The terminal guidance mode used for this phase consists of two feedback loops depicted in the block diagram of Figure 3 . The outer guidance loop provides command signals to the vehicle attitude control system and the inner control loop provides vehicle torques to follow the commands.

The asteroid flyby guidance loop is designed to generate tracking signals for the vehicle to follow. This loop incorporates a sixstate Kalman filter using measurement information from the UV/VIS camera and the LIDAR camera to estimate relative position and velocity between spacecraft and asteroid in the inertial frame. The camera-based measurements occur at $21 / 2$ second increments, with a constantvelocity model assumed between measurements. The six states are then converted to the body frame, using the estimated attitude, and commanded body rates and quaternions are generated.

The inner attitude control loop is designed to follow the commanded body rates and quaternions from the guidance loop. The reaction wheels are used during most of the flyby phase, with thrusters being engaged during the critical five-minute period of closest approach. The thruster on/off logic consists of simple bang-bang control with a deadband of 0.03 degrees.

During all reaction wheel activities momentum management was active to prevent saturation of the reaction wheels. The momentum management system was designed to autonomously monitor reaction wheel speeds, determine if a momentum dump was required, and to set up momentum dumping thruster pulses. An option was available to trigger a momentum dump if desired. With the mission orbits as selected, momentum management was expected to be used every few days during lunar mapping.

\section{Component Selection and Hardware Description}

Typically for this type of mission, a narrow field-of-view star tracker is used in combination with high performance low drift gyros for fine pointing, with the required coarse sensors for initial attitude acquisition and transition into fine pointing control. For Clementine, the ADCS design had to take advantage of new lightweight systems to minimize required hardware and keep costs low. A system was selected to rely on a wide field-ofview, lightweight ST camera that could provide three-axis attitude determination with a single image, a low performance IMU for attitude propagation between frequent ST camera updates, and a set of lightweight reaction wheels as actuators.

The ST camera was one of the primary payload sensors to be tested by this mission and was provided by LLNL. This small, lightweight, wide field-of-view camera provided full three-axis lost-in-space attitude determination with a single image. The sensor sends the image to a sensor image processor which then identifies the brightest objects and creates triangles which are then matched with an on-board star catalogue. An attitude quaternion is then generated and reported to the attitude determination code. Two ST cameras with no overlapping coverage were used to ensure attitude updates throughout the mission. An earlier version of this camera and different software had been used previously for a few days in low earth orbit. The processing of the star tracker image and determination of the attitude quaternion based on the image was performed in the payload sensor image processor. The ability to execute the quaternion generation software on the housekeeping computer was available if the payload sensor image processor was not available. Performance characteristics of the ST camera are given in Table 5.

Two small lightweight IMUs, neither of which have flown in space, were selected. The Litton LN200 IMU, using Interferometric Fiber Optic Gyros and Silicon Accelerometers, and the Honeywell Lightweight Advanced Implementation Technology IMU, using Ring Laser Gyros and Sundstrand RBA-500 Accelerometers, were selected. Both IMUs were active and providing incremental angles and 
Table 5: LLNL ST Camera

\begin{tabular}{l|c}
\hline \hline Characteristic & Value \\
\hline Mass & $364 \mathrm{~g}$ \\
Size & $4.6 \mathrm{in} \times 4.6 \mathrm{in} \times 5.2 \mathrm{in}$ \\
Power Dissipation & $4.5 \mathrm{~W}$ \\
Spectral Range & $0.4-1.1 \mu \mathrm{m}$ \\
Field Of View & $43.2 \times 28.4 \mathrm{deg}$ \\
IFOV & $1.28 \mathrm{mrad}(0.073 \mathrm{deg})$ \\
Accuracy & $150 \times 150 \times 450 \mu \mathrm{rad}$ \\
Number Of Pixels & $576 \times 384$ \\
Integration Time & $200 \mathrm{msec}$ \\
Sensitivity & SNR $=20 \mathrm{at} \mathrm{Mv}=4.5$ \\
\hline \hline
\end{tabular}

velocities. Data checking was performed on the output of both units prior to input to the attitude determination software algorithms. Specification requirements for the Clementine IMUs are shown in Table 6.

Table 6: Clementine IMU Specification

\begin{tabular}{l|c|}
\hline \hline Characteristic & Value \\
\hline Mass & $<1 \mathrm{~kg}$ \\
Volume & $<50 \mathrm{cu}-\mathrm{in}$ \\
Power & $<10 \mathrm{~W}$ \\
Gyro Max Rate & $+/-600 \mathrm{deg} / \mathrm{sec}$ \\
Gyro Bias Accuracy & $1 \mathrm{deg} / \mathrm{hr}$ \\
Gyro Scale Factor Accuracy & $100 \mathrm{ppm}$ \\
Gyro Random Walk & $0.25 \mathrm{deg} / \mathrm{rt}-\mathrm{hr}$ \\
Gyro Noise & $1.5 \mathrm{arcsec}$ \\
Gyro LSB & $5 \mathrm{arcsec}$ \\
Accel Max Acceleration & $+/-30 \mathrm{~g}$ \\
Accel Bias Accuracy & $10 \mathrm{mg}$ \\
Accel Scale Factor Accuracy & $100 \mathrm{ppm}$ \\
Accel Noise & $1 \mathrm{~mm} / \mathrm{sec}$ \\
Accel LSB & $0.003 \mathrm{ft} / \mathrm{sec}$ \\
\hline \hline
\end{tabular}

Four small Ball Aerospace Reaction Wheels (RW) with internal electronics were used as actuators for the three-axis control system. The previous version of this reaction wheel system used external electronics to drive the four wheels. The reaction wheels are mounted such that three are orthogonal and a fourth is a skewed backup. The RW characteristics are provided in Table 7.

Table 7: Reaction Wheel Performance

\begin{tabular}{l|c|}
\hline \hline Characteristic & Value \\
\hline Total Mass (4 Wheels) & $11.2 \mathrm{~kg}$ \\
Volume Per Wheel & $150 \mathrm{cu}-$-in \\
Steady-State Power (3 Active) & $28 \mathrm{~W}$ \\
Peak Power (3 Active) & $64 \mathrm{~W}$ \\
Momentum Storage & $2 \mathrm{Nms}$ \\
Reaction Torque & $6 \mathrm{oz}-\mathrm{in}$ \\
Speed Range & $+/-2500 \mathrm{rpm}$ \\
\hline \hline
\end{tabular}

The ST Cameras, IMUs, \& RWs had no space flight experience in their as-built configurations. Earlier versions of the STCs and the RWs each had one week of space flight experience. The total system weight of this hardware, see Table 8, is under $13 \mathrm{Kg}$.

Table 8: Total ADCS Weight

\begin{tabular}{l|c|}
\hline \hline Component & Mass (kg) \\
\hline 2 Star Tracker Cameras: & 0.6 \\
2 Inertial Measurement Units: & 1.2 \\
4 Reaction Wheels: & 11.1 \\
\hline System Total: & 12.9 \\
\hline \hline
\end{tabular}

\section{Attitude Determination and Control System Testing}

To meet the required launch date and to allow sufficient time for hardware delivery and software development, it was necessary to compress the hardware/software integration and test phase of the program. Even though compressed, the testing phase was still required to ensure proper functionality, performance, and flight readiness of hardware and software. To meet the demands of the schedule, the amount of documentation required by the program for hardware and software testing was limited to sufficient test procedure and test report detail to ensure the flight readiness of the hardware and software. The hardware testing consisted of initial hardware/software integration tests with the vehicle, performance testing, and functional testing throughout the system level environmental testing phase. The software testing started first at the algorithm level with detailed simulation by the ADCS subsystem engineers, followed by code testing by the software group, followed by test bed simulation of the full system running through mission sequences on the operational test bed. The operational test bed contained, an actual flight computer, hardware/software simulations of the IMUs, RWs, and STCs, and a spacecraft dynamic model simulation. Control system performance comparisons were run between the subsystem detailed simulations and the top level operational test bed for all of the major mission operations. The dynamic representations of the IMUs, RWs, and STCs were updated based on data collected from functional tests performed on the ground with the actual flight hardware.

Hardware testing consisted of a series of functional tests to verify proper operation of hardware. These tests were performed after integration with the vehicle, before, during, and after environmental testing, and prior to launch. ADCS testing of hardware consisted of functional 
testing on the ST cameras, IMUs, and RWs. The ST camera functional testing consisted of taking pictures of a simulated star field with different orientations of the star field in the sensor field-ofview to confirm operation and sign convention. Outdoor tests were performed twice, once early in the integration cycle to determine the alignment of the internal ST camera axes with the external case and a final end-to-end test was performed at Vandenberg to confirm the alignment of the ST camera axes to the spacecraft axes. The IMU funtional testing consisted of earth rate measurements and simple body rotations. The noise level in the IMUs was monitored for signs of degradation through periodic static tests. The $\mathrm{RW}$ functional testing consisted of tests to confirm spin direction and tests to observe spin-up and spin-down characteristics and breakaway friction.

Software testing consisted of a significant number of subsystem simulation runs followed by operational test bed simulations to verify proper implementation of the algorithms. The primary tools to verify performance were the detailed subsystem simulations. Functional tests were performed on the flight hardware to verify commanding of the reaction wheels for the reaction wheel control mode and the thrusters for the thruster control modes.

\section{Conclusions}

In this paper we described the Clementine ADCS design which was capable of meeting all the requirements of a very difficult and complex mission involving both prolate spinning and threeaxis stabilized configurations. The design, hardware procurement, test, and integration were all performed within two years of program start. New lightweight attitude determination and control system components consisting of a new wide field-of-view lost-in-space star tracker camera, two relatively low performance IMUs, and a new lightweight reaction wheel with internal drive electronics were qualified and flown in space.

\section{Acknowledgments}

The authors wish to thank Mr. Robert Stapleford of Space Applications Corporation in El Segundo, CA and his staff for their support of the ADCS design and Mr. Samuel Hollander of the Naval Research Laboratory for his review of the manuscript.

\section{References}

${ }^{1}$ Rustan, P.L., "Clementine: Mining New Uses for SDI Technology," Aerospace America, Jan. 1994, pp. 38-41.

2Regeon, P.A., Chapman, R.J., and Baugh, R., "Clementine - The Deep Space Program Science Experiment (DSPSE)," IAA International Conference on Low-Cost Planetary Missions, The Johns Hopkins University Applied Physics Laboratory, Laurel, MD, April 12-15, 1994.

${ }^{3}$ Kaufman, B., Middour, J., Dasenbrock, R., and Campion, R., "The Deep Space Program Science Experiment Mission: Astrodynamics Mission Planning," 44th Congress of the International Astronautical Federation, Graz, Austria, Oct. 16-22, 1993. 